\title{
Protein identification by mass spectrometry
}

- SDS-PAGE fractionated proteins were reduced, alkylated with iodoacetamide, and in-gel digested with side-chain protected porcine trypsin (http://ms-facility.ucsf.edu/ingel.html)

- An aliquot of each digest was fractionated by reversed phase HPLC using a Ultimate pump/Famos autosampler system (Dionex/LC Packings), a homemade nanocolumn $(C 12,75 \mu \mathrm{m} \times 150 \mathrm{~mm})$ at a flow rate of $\sim 300$ $\mathrm{nL} / \mathrm{min}$; developing a 5\% B-50 \% B linear gradient over $30 \mathrm{~min}(01 \%$ formic acid/water/acetonitrile); and using an LTQ iontrap (Thermo Finnigan) as the on-line detector in a data-dependent, "triple play" (full scan/zoom-in/CID on multiply charged ions) fashion.

- Datafiles were processed with Mascot distiller, and database searches were performed using an in-house Mascot server. The full SwissProt database was searched; only tryptic cleavages were considered; 2 missed cleavages were permitted; Cys-carbamidomethylation was considered as fixed modification, while Met-oxidation, pyroglutamic acid formation from $\mathrm{N}$-terminal $\mathrm{Gln}$, and the acetylation of the $\mathrm{N}$ terminus of the protein were the considered variable modifications. Mass accuracy for precurzor ions: $2 \mathrm{Da}$; for CID fragments: 0.8 Da. 


\section{Figure legend}

Database search results for one of the identified p97-peptides. Interestingly this sequence represents the post-translationally modified N-terminus of the protein: Met-1 was replaced by an acetyl group.

a) CID data for $m / z 845.8(2+)$, fragments assigned by Mascot

b) Potential fragments for the identified peptide. All the red ones were observed in the CID experiment 


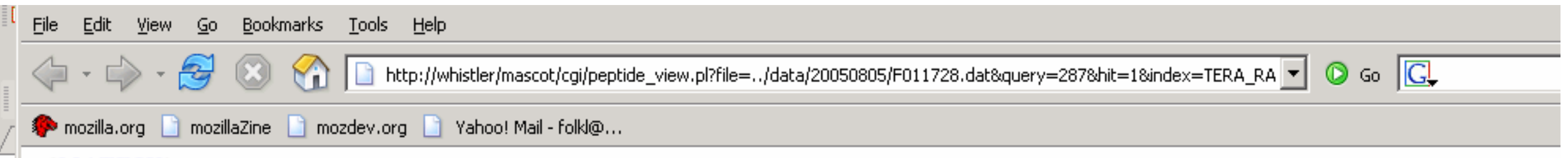

\section{$\left\{\begin{array}{l}M A T R I X \\ S C I E N C E\end{array}\right\}$ Mascot Search Results}

\section{Peptide View}

MSMS Fragmentation of ASGADSKGDDLSTALK

Found in TERA_RAT, (P46462) Transitional endoplasmic reticulum ATPase (TER ATPase) (15S Mg(2+)-ATPase p97 subunit) (Va

Match to Query 287: 1689.621748 from(845.818150,2+)

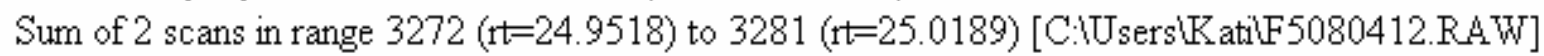

From data file C:DOCUME $1 \mathrm{~K}$ atiLOCALS 1Temp Dis 16A.tmp

Click mouse within plot area to zoom in by factor of two about that point

Or, Plotfrom $\sqrt{200}$ to $\longdiv { 1 6 0 0 } \mathrm { Da }$

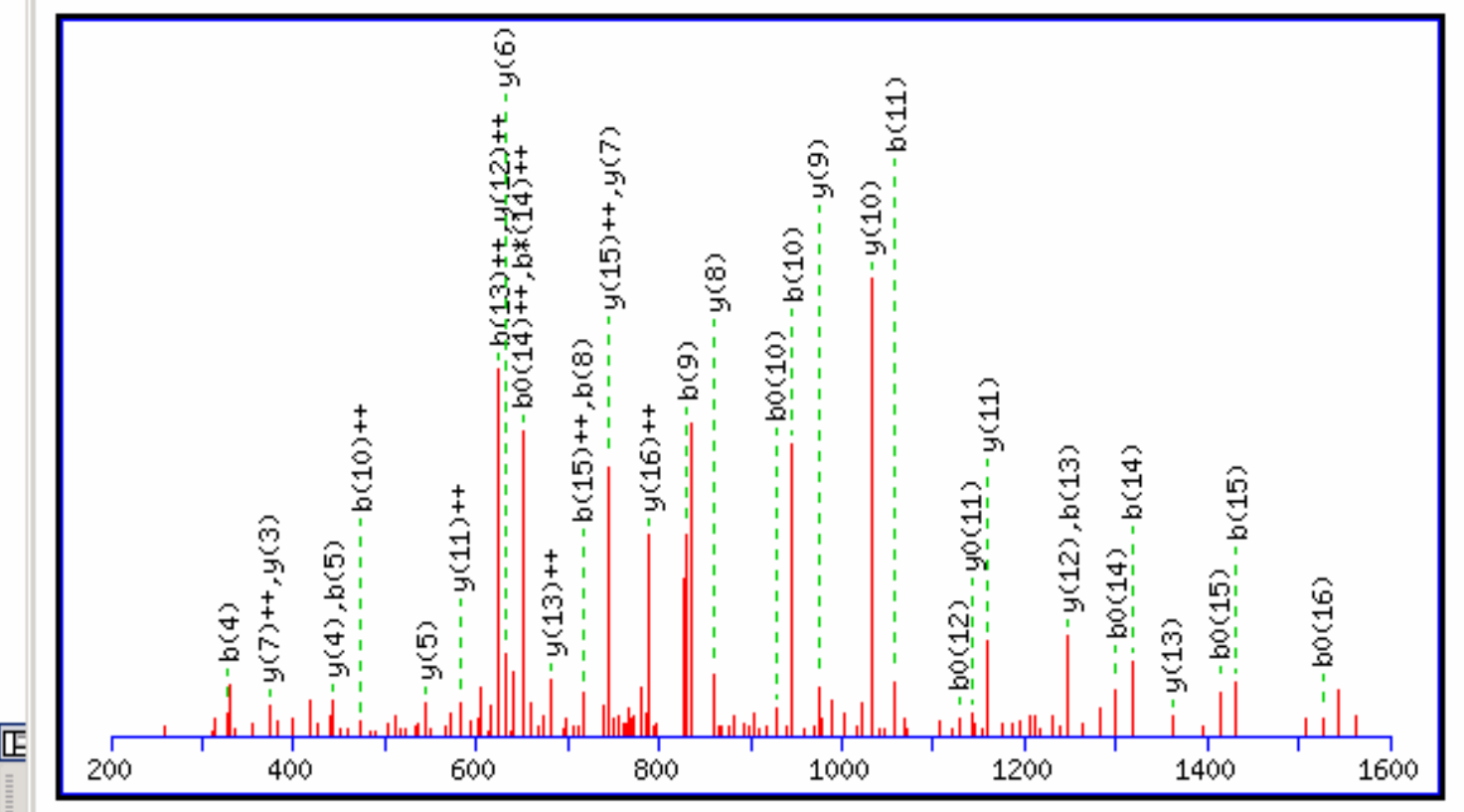


mozilla.org $\square$ mozillazine $\square$ mozdev.org $\square$ Yahoo! Mail - folk@...

Monoisotopic mass of neutral peptide Mr(calc): 1689.84

Fixed modifications: Carbamidomethyl (C)

Variable modifications:

H-term : N-Acetyl (Protein)

Ions Score: 87 Expect: $3.5 \mathrm{e}-06$

Matches (Bold Red): $37 / 170$ fragment ions using 42 most intense peaks

\begin{tabular}{|c|c|c|c|c|c|c|c|c|c|c|c|c|c|c|}
\hline$\#$ & b & $\mathbf{b}^{++}$ & $\mathbf{b}^{\star}$ & $\mathbf{b}^{\star^{++}}$ & $\mathbf{b}^{0}$ & $\mathbf{b}^{0++}$ & Seq. & $\mathbf{y}$ & $y^{++}$ & $\mathbf{y}^{*}$ & $y^{\star^{++}}$ & $y^{0}$ & $y^{0++}$ & $\#$ \\
\hline 1 & 114.05 & 57.53 & & & & & A & & & & & & & 17 \\
\hline 2 & 201.09 & 101.05 & & & 183.08 & 92.04 & $S$ & 1577.80 & 789.40 & 1560.78 & 780.89 & 1559.79 & 780.40 & 16 \\
\hline 3 & 258.11 & 129.56 & & & 240.10 & 120.55 & $\mathrm{G}$ & 1490.77 & 745.89 & 1473.74 & 737.38 & 1472.76 & 736.88 & 15 \\
\hline 4 & 329.15 & 165.08 & & & 311.13 & 156.07 & $\mathrm{~A}$ & 1433.75 & 717.38 & 1416.72 & 708.86 & 1415.74 & 708.37 & 14 \\
\hline 5 & 444.17 & 222.59 & & & 426.16 & 213.58 & D & 1362.71 & 681.86 & 1345.68 & 673.35 & 1344.70 & 672.85 & 13 \\
\hline 6 & 531.20 & 266.11 & & & 513.19 & 257.10 & $S$ & 1247.68 & 624.35 & 1230.66 & 615.83 & 1229.67 & 615.34 & 12 \\
\hline 7 & 659.30 & 330.15 & 642.27 & 321.64 & 641.29 & 321.15 & $\mathrm{~K}$ & 1160.65 & 580.83 & 1143.63 & 572.32 & 1142.64 & 571.82 & 11 \\
\hline 8 & 716.32 & 358.66 & 699.29 & 350.15 & 698.31 & 349.66 & $\mathrm{G}$ & 1032.56 & 516.78 & 1015.53 & 508.27 & 1014.55 & 507.78 & 10 \\
\hline 9 & 831.35 & 416.18 & 814.32 & 407.66 & 813.34 & 407.17 & D & 975.54 & 488.27 & 958.51 & 479.76 & 957.53 & 479.27 & 9 \\
\hline 10 & 946.37 & 473.69 & 929.35 & 465.18 & 928.36 & 464.69 & $\mathrm{D}$ & 860.51 & 430.76 & 843.48 & 422.24 & 842.50 & 421.75 & 8 \\
\hline 11 & 1059.46 & 530.23 & 1042.43 & 521.72 & 1041.45 & 521.23 & $\mathrm{~L}$ & 745.48 & 373.24 & 728.46 & 364.73 & 727.47 & 364.24 & 7 \\
\hline 12 & 1146.49 & 573.75 & 1129.46 & 565.24 & 1128.48 & 564.74 & $S$ & 632.40 & 316.70 & 615.37 & 308.19 & 614.39 & 307.70 & 6 \\
\hline 13 & 1247.54 & 624.27 & 1230.51 & 615.76 & 1229.53 & 615.27 & $\mathrm{~T}$ & 545.37 & 273.19 & 528.34 & 264.67 & 527.36 & 264.18 & 5 \\
\hline 14 & 1318.58 & 659.79 & 1301.55 & 651.28 & 1300.57 & 650.79 & $\mathrm{~A}$ & 444.32 & 222.66 & 427.29 & 214.15 & & & 4 \\
\hline 15 & 1431.66 & 716.33 & 1414.63 & 707.82 & 1413.65 & 707.33 & I & 373.28 & 187.14 & 356.25 & 178.63 & & & 3 \\
\hline 16 & 1544.74 & 772.88 & 1527.72 & 764.36 & 1526.73 & 763.87 & L & 260.20 & 130.60 & 243.17 & 122.09 & & & 2 \\
\hline 17 & & & & & & & $\mathrm{~K}$ & 147.11 & 74.06 & 130.09 & 65.55 & & & 1 \\
\hline
\end{tabular}




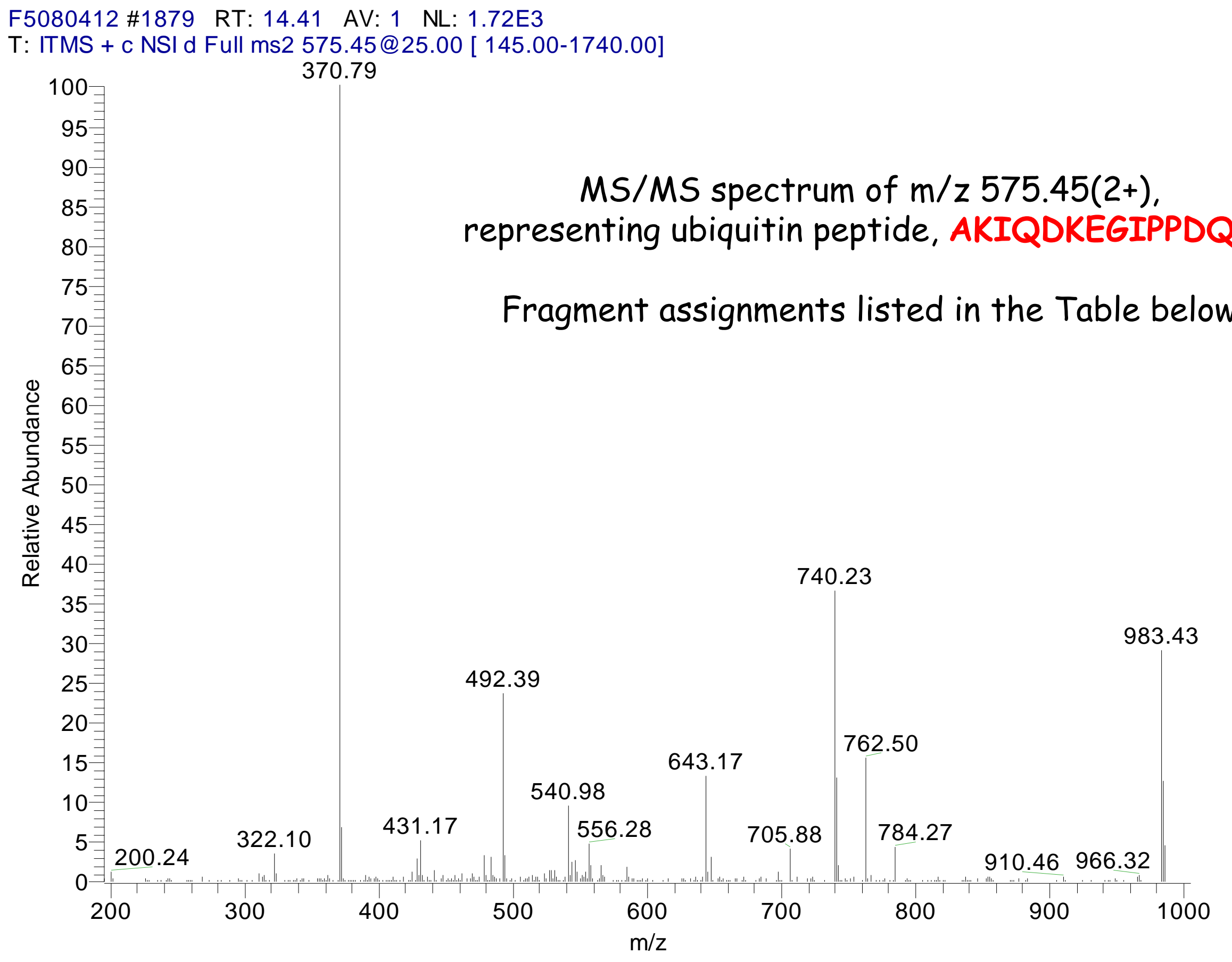




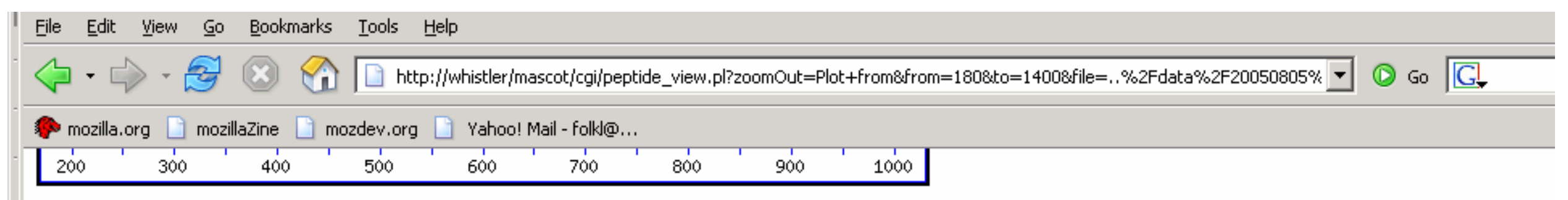

Monoisotopic mass of neutral peptide Mr(calc): 1721.91

Fixed modifications: Carbamidomethyl (C)

Ions Score: 53 Expect : 0.0083

Matches (Bold Red): $39 / 152$ fragment ions using 37 most intense peaks

\begin{tabular}{|c|c|c|c|c|c|c|c|c|c|c|c|c|c|c|}
\hline$\#$ & b & $\mathbf{b}^{++}$ & $\mathbf{b}^{*}$ & $b^{\star^{++}}$ & $\mathbf{b}^{0}$ & $b^{0++}$ & Seq. & $\mathbf{y}$ & $y^{++}$ & $\mathbf{y}^{*}$ & $y^{\star^{++}}$ & $y^{0}$ & $\mathbf{y}^{0++}$ & $\#$ \\
\hline 1 & 72.04 & 36.53 & & & & & A & & & & & & & 15 \\
\hline 2 & 200.14 & 100.57 & 183.11 & 92.06 & & & $\mathrm{~K}$ & 1651.88 & 826.44 & 1634.85 & 817.93 & 1633.87 & 817.44 & 14 \\
\hline 3 & 313.22 & 157.12 & 296.20 & 148.60 & & & I & 1523.78 & 762.39 & 1506.75 & 753.88 & 1505.77 & 753.39 & 13 \\
\hline 4 & 441.28 & 221.14 & 424.26 & 212.63 & & & $Q$ & 1410.70 & 705.85 & 1393.67 & 697.34 & 1392.69 & 696.85 & 12 \\
\hline 5 & 556.31 & 278.66 & 539.28 & 270.14 & 538.30 & 269.65 & D & 1282.64 & 641.82 & 1265.61 & 633.31 & 1264.63 & 632.82 & 11 \\
\hline 6 & 684.40 & 342.71 & 667.38 & 334.19 & 666.39 & 333.70 & $\mathrm{~K}$ & 1167.61 & 584.31 & 1150.59 & 575.80 & 1149.60 & 575.30 & 10 \\
\hline 7 & 813.45 & 407.23 & 796.42 & 398.71 & 795.44 & 398.22 & $\mathbf{E}$ & 1039.52 & 520.26 & 1022.49 & 511.75 & 1021.51 & 511.26 & 9 \\
\hline 8 & 870.47 & 435.74 & 853.44 & 427.22 & 852.46 & 426.73 & $\mathrm{G}$ & 910.47 & 455.74 & 893.45 & 447.23 & 892.46 & 446.74 & 8 \\
\hline 9 & 983.55 & 492.28 & 966.53 & 483.77 & 965.54 & 483.27 & I & 853.45 & 427.23 & 836.43 & 418.72 & 835.44 & 418.22 & 7 \\
\hline 10 & 1080.60 & 540.81 & 1063.58 & 532.29 & 1062.59 & 531.80 & $\mathbf{P}$ & 740.37 & 370.69 & 723.34 & 362.17 & 722.36 & 361.68 & 6 \\
\hline 11 & 1177.66 & 589.33 & 1160.63 & 580.82 & 1159.65 & 580.33 & $\mathbf{P}$ & 643.32 & 322.16 & 626.29 & 313.65 & 625.31 & 313.16 & 5 \\
\hline 12 & 1292.68 & 646.85 & 1275.66 & 638.33 & 1274.67 & 637.84 & $\mathrm{D}$ & 546.26 & 273.64 & 529.24 & 265.12 & 528.25 & 264.63 & 4 \\
\hline 13 & 1420.74 & 710.88 & 1403.72 & 702.36 & 1402.73 & 701.87 & $Q$ & 431.24 & 216.12 & 414.21 & 207.61 & & & 3 \\
\hline 14 & 1548.80 & 774.90 & 1531.78 & 766.39 & 1530.79 & 765.90 & $Q$ & 303.18 & 152.09 & 286.15 & 143.58 & & & 2 \\
\hline 15 & & & & & & & $\mathbf{R}$ & 175.12 & 88.06 & 158.09 & 79.55 & & & 1 \\
\hline
\end{tabular}

曾 0.5 年- - . 
F5080412 \#3368 RT: 25.68 AV: 1 NL: 4.90E1

T: ITMS + c NSI d Full ms2 633.05@25.00 [ 160.00-1280.00]

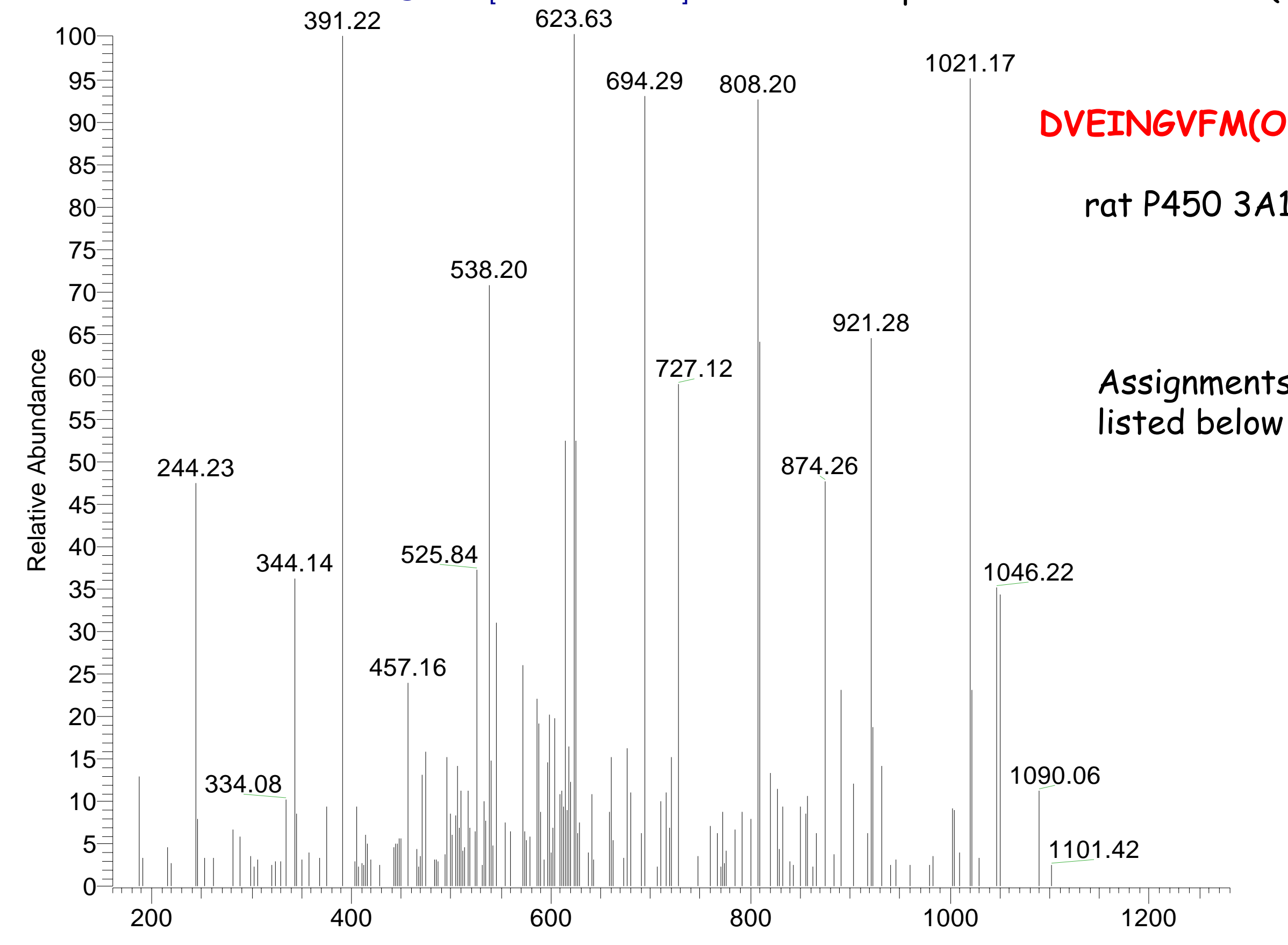

MS/MS spectrum of $\mathrm{m} / \mathrm{z} 632.72(2+)$ 


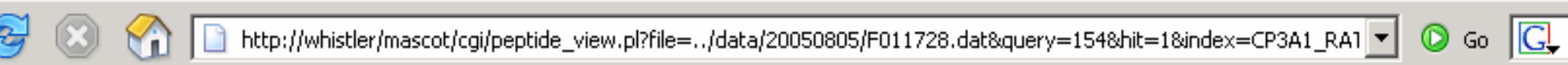

mozilla.org $\square$ mozillazine $\square$ mozdev.org $\square$ Yahoo! Mail - folk@...

Monoisotopic mass of neutral peptide Mr(calc): 1263.62

Fixed modifications: Carbamidomethyl (C)

Variable modifications :

M9 : Oxidation (M)

Ions Score: 58 Expect: 0.0027

Matches (Bold Red): $16 / 96$ fragment ions using 20 most intense peaks

\begin{tabular}{|c|c|c|c|c|c|c|c|c|c|c|c|c|c|c|}
\hline$\#$ & $\mathbf{b}$ & $b^{++}$ & $\mathbf{b}^{\star}$ & $b^{\star^{++}}$ & $\mathbf{b}^{0}$ & $b^{0++}$ & Seq. & y & $y^{++}$ & $\mathrm{y}^{*}$ & $y^{\star^{++}}$ & $y^{0}$ & $y^{0++}$ & $\#$ \\
\hline 1 & 116.03 & 58.52 & & & 98.02 & 49.52 & $\mathrm{D}$ & & & & & & & 11 \\
\hline 2 & 215.10 & 108.05 & & & 197.09 & 99.05 & $\mathrm{~V}$ & 1149.60 & 575.30 & 1132.57 & 566.79 & 1131.59 & 566.30 & 10 \\
\hline 3 & 344.15 & 172.58 & & & 326.13 & 163.57 & E & 1050.53 & 525.77 & 1033.50 & 517.25 & 1032.52 & 516.76 & 9 \\
\hline 4 & 457.23 & 229.12 & & & 439.22 & 220.11 & I & 921.49 & 461.25 & 904.46 & 452.73 & & & 8 \\
\hline 5 & 571.27 & 286.14 & 554.25 & 277.63 & 553.26 & 277.13 & $\mathrm{~N}$ & 808.40 & 404.70 & 791.38 & 396.19 & & & 7 \\
\hline 6 & 628.29 & 314.65 & 611.27 & 306.14 & 610.28 & 305.65 & $\mathrm{G}$ & 694.36 & 347.68 & 677.33 & 339.17 & & & 6 \\
\hline 7 & 727.36 & 364.18 & 710.34 & 355.67 & 709.35 & 355.18 & $\mathrm{~V}$ & 637.34 & 319.17 & 620.31 & 310.66 & & & 5 \\
\hline 8 & 874.43 & 437.72 & 857.40 & 429.21 & 856.42 & 428.71 & $\mathrm{~F}$ & 538.27 & 269.64 & 521.24 & 261.13 & & & 4 \\
\hline 9 & 1021.47 & 511.24 & 1004.44 & 502.72 & 1003.46 & 502.23 & M & 391.20 & 196.10 & 374.17 & 187.59 & & & 3 \\
\hline 10 & 1118.52 & 559.76 & 1101.49 & 551.25 & 1100.51 & 550.76 & $\mathbf{P}$ & 244.17 & 122.59 & 227.14 & 114.07 & & & 2 \\
\hline 11 & & & & & & & $\mathrm{~K}$ & 147.11 & 74.06 & 130.09 & 65.55 & & & 1 \\
\hline
\end{tabular}

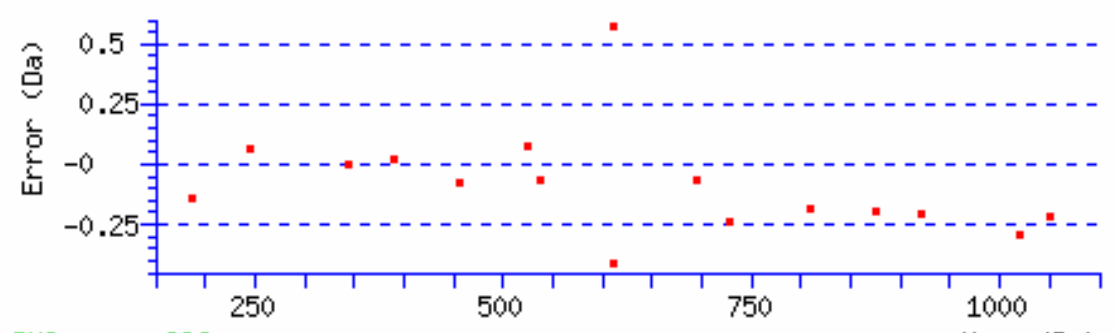

Rits error 386 ppm

Mass (Da) 